\title{
Perfil mineral en bovinos lecheros de Santa Fe, Argentina
}

\author{
Luna, M.L.; Roldan, V.P.
}

Cátedras de Química I y II, Facultad de Ciencias Veterinarias, Universidad Nacional del Litoral, P. Kreder 2805, Esperanza (3080), Santa Fe (Argentina). Tel (03496)-420639. E-mail: vroldan@fcv.unl.edu.ar.

\begin{abstract}
Resumen
Luna, M.L.; Roldan, V.P.: Perfil mineral en bovinos lecheros de Santa Fe, Argentina. Rev. vet. 24: 1, 47-52, 2013. Los minerales son nutrientes esenciales que representan el $5 \%$ del peso vivo del bovino. Sus deficiencias y desequilibrios afectan la producción, reproducción y salud animal. Para indagar el estatus mineral del ganado Holstein y cruza Holstein-Friesian en tres establecimientos del Departamento Las Colonias (Santa Fe, Argentina), se investigaron sangre y leche de 15 vacas en 4 períodos fisiológicos, durante las primaveras y veranos de los años 2006 a 2008. Los bovinos se alimentaron con recursos pastoriles, en condiciones edáficas diferentes. En las muestras de suero de los tres campos se evidenció hipocalcemia significativa $(\mathrm{p}<0,05)$ en gestación y lactación, relacionada con las exigencias fisiológicas y productivas; hiponatremia durante el período de lactación, debido a la mayor demanda de mineral para la secreción de leche, e hipomagnesemia $(\mathrm{p}<0,05)$ debida al estrés del animal próximo al parto y a la pérdida del mineral por sudoración en el verano. Los valores bajos significativos $(\mathrm{p}<0,05)$ de cinc en suero durante la gestación tendrían relación con la etapa invernal. En las muestras de leche de los distintos campos, se registraron disminuciones de los valores de cinc, magnesio, calcio y potasio $(\mathrm{p}<0,05)$ hacia los 60 días del inicio de la lactancia, que se podrían justificar por la depleción de minerales hacia la leche. No se verificaron interferencias entre cobre y molibdeno en suero. Se espera que los resultados obtenidos brinden utilidad a los profesionales y productores tamberos de la zona.
\end{abstract}

Palabras clave: bovino lechero, macrominerales, oligoelementos, nutrición, Argentina.

\begin{abstract}
Luna, M.L.; Roldan, V.P.: Mineral profile in dairy cows from Santa Fe, Argentina. Rev. vet. 24: 1, 47-52, 2013. Minerals are essential nutrients that represent $5 \%$ of the bovine liveweight. Lacks and disorders of minerals in animal nutrition affect the production, reproduction and health. In this assay, blood and milk were analyzed in 15 Holstein and Holstein-Friesian cows in four physiological states of three dairy farms with natural pastures from Las Colonias (Santa Fe, Argentina) during the spring and summer of 2006-2008. Poor edaphic conditions and low historical mineral composition of the soil characterized the area. Analysis of sera samples showed the following results: significant $(\mathrm{p}<0,05)$ Ca-deficiency related to physiological and productive requirements; hyponatraemia during the lactation period due to increasing demand for milk production; and during gestation hypomagnesaemia $(\mathrm{p}<0,05)$ due to stress of animals prior to birth and mineral loss through sweat during the summer. Significant $(p<0,05)$ low values of serum zinc during pregnancy would be related to low temperature months. The analysis of milk samples from the dairy farms showed significantly $(p<0,05)$ decreased average values of zinc, magnesium, calcium and potassium towards day 60 of lactation, which may be explained due to the utilization of minerals for milk production. The high concentrations of zinc in the grass antagonizes with the presence of high iron and molybdenum concentrations in the diet, condition observed in a dairy farm. We expect that our results may offer useful information to veterinarians and farmers of the considered geographical area.
\end{abstract}

Key words: dairy cow, macrominerals, oligoelements, nutrition, Argentina.

\section{INTRODUCCIÓN}

Las deficiencias y desequilibrios de minerales en la nutrición animal afectan la producción, reproduc-

Recibido: 1 agosto 2012 / Aceptado: 8 febrero 2013

Este trabajo es parte de la tesis de la Bioq. Mónica L. Luna,

Maestría en Ciencias Veterinarias, FCV-Univ.Nac.Litoral. ción y salud de los rodeos al alterar la funcionalidad de biomoléculas y tejidos, el metabolismo y la fertilidad. Los minerales son nutrientes esenciales que represen$\tan$ aproximadamente el 5\% del peso vivo del bovino. Han sido reconocidos más de veintiséis elementos que pueden ser clasificados en macro y microelementos $u$ oligoelementos $^{3}$. 
Macroelementos son los minerales que los animales requieren en cantidades del orden de g/día o como porcentaje de materia seca (MS) consumida en la ración; entre ellos se encuentran: calcio, magnesio, sodio, potasio y otros. Estos minerales se distribuyen en mayor proporción en los tejidos de sostén, contribuyen al mantenimiento de las propiedades fisicoquímicas del ambiente ruminal (capacidad buffer, presión osmótica y tasa de dilución), son componentes celulares y activadores enzimáticos, imprescindibles para mantener las funciones vitales ${ }^{1}$.

Las necesidades de oligoelementos se expresan en $\mathrm{mg} / \mathrm{día}$ o en ppm (partes por millón) de MS. Entre ellos se encuentran cobre, cinc, hierro y otros. Cumplen funciones de activadores o cofactores enzimáticos del tipo iones metálicos, forman parte de las hormonas, participan del transporte de oxígeno, regulan reacciones enzimáticas microbianas a nivel ruminal y juegan un rol importante en el mantenimiento del desarrollo fetal, la función reproductiva y la actividad inmunitaria ${ }^{1}$.

Las deficiencias se clasifican en primaria, cuando es insuficiente la cantidad disponible en la dieta, y secundaria o condicionada, cuando ocurre por interacción o interferencia de otros elementos presentes en el alimento, que impiden la correcta absorción del mineral ${ }^{8,20}$. La interacción negativa es uno de los principales factores dietarios que causan baja biodisponibilidad de los nutrientes minerales. Las interacciones de importancia nutricional incluyen las del tipo cinc-cobre, molibdenocobre y hierro-cobre. Si el molibdeno está presente en alta concentración, tendrá un efecto antagónico sobre la absorción del cobre presente en la dieta ${ }^{15}$. Dado que los pastos obtienen sus constituyentes del suelo donde crecen, la calidad de los alimentos estará comprometida si existen deficiencias minerales en el suelo ${ }^{9}$.

A medida que la gestación avanza, aumentan las demandas de nutrientes y, concomitantemente, disminuye el consumo voluntario. La transición a la lactancia es el período que se extiende desde las tres semanas previas hasta las tres semanas posteriores al parto ${ }^{13}$, etapa durante la cual se incrementan sustancialmente los trastornos nutricionales clínicos y subclínicos, como por ejemplo la hipocalcemia. Por otra parte, la lactancia desencadena una serie de cambios fisiológicos propios de un estado de homeorresis, durante el cual el organismo se aparta temporalmente del estado de homeostasis y desvía los nutrientes para satisfacer un estado fisiológico determinado.

En Argentina fueron detectadas deficiencias de sodio en las provincias de Corrientes, Chaco, Formosa y Misiones. Además, en el este de Chaco y Formosa se encontraron niveles subnormales de magnesio en muestras de forrajes y sueros bovinos, así como deficiencias de cinc en muestras de suelo, pasturas, sangre e hígado de bovinos. En las provincias de Corrientes, Chaco, Formosa, norte de Entre Ríos, Misiones, Salta, Santiago del Estero y en los bajos submeridionales de Santa $\mathrm{Fe}$, la indisponibilidad de cobre provocó carencia primaria de este oligoelemento, agravada por el exce- so de molibdeno en pastos y de azufre en agua, o por exceso de hierro en forrajes ${ }^{8}$. En el nordeste argentino, las zonas más afectadas por el déficit de selenio fueron el norte y sur de Santa Fe, Chaco, Formosa, Santiago del Estero y Entre Ríos ${ }^{8}$. En Córdoba se detectaron carencias de zinc, magnesio, calcio y selenio, este último inculpado por provocar baja fertilidad en el ganado ${ }^{11}$. En la Provincia de Santa Fe, las carencias de calcio y fósforo se atribuyen al incremento de la producción ${ }^{3}$.

Para la región central de Santa Fe, Argentina, no se dispone de datos actualizados sobre el contenido de minerales en fluidos biológicos de vacas lecheras. Así, se estimó relevante caracterizar el perfil mineral en sangre y leche en distintas etapas del periodo productivo $y$ en pasturas de alfalfa y verdeos de invierno-verano, con el objetivo de conocer los déficits minerales existentes y -en caso necesario- suplementar para prevenir el impacto negativo que pudieran generar en la salud, producción y reproducción.

\section{MATERIAL Y MÉTODOS}

Se recolectaron muestras de sangre y leche de bovinos de la raza Holstein y de la cruza HolsteinFriesian en distintos estados fisiológicos, durante las temporadas de primavera y verano de 2006 a 2008. Las vacas fueron seleccionadas al azar, en tres establecimientos de la región centro de Santa Fe (Departamento Las Colonias), de diferentes condiciones edáficas. Los animales se alimentaron con alfalfa como principal componente de la dieta y forrajes verdes en invierno y verano, sin suplementación mineral.

Los estados fisiológicos estudiados fueron gestación (dos meses previos a la parición), preparto (15 días antes de la parición), posparto (15 días posteriores a la parición) y lactación avanzada (2 meses posteriores a la fecha de parto). Cada establecimiento fue identificado con letras: campo A (Escuela de Agricultura, Ganadería y Granja de las facultades de Veterinaria y Agronomía, Universidad Nacional del Litoral), campo B (Escuela Agrotécnica No 299 "Carlos Sylvestre Begnis") y campo C (establecimiento agropecuario "Mundo Nuevo"). A lo largo de los cuatro períodos fisiológicos siempre se muestrearon los mismos animales $(n=15)$. Se hace constar que el campo A había sufrido los efectos de una inundación en el año 2003.

La sangre fue obtenida por venopunción yugular. En las muestras de suero, se destruyó la materia orgánica $\left(1 \mathrm{ml}\right.$ de suero a $90^{\circ} \mathrm{C}$ y $5 \mathrm{ml} \mathrm{HNO}_{3}$ concentrado, 2 horas). Los minerales se determinaron respetando las técnicas propuestas por la $\mathrm{AOAC}^{2}$.

La leche se obtuvo por ordeño manual y se llevó a sequedad en una estufa a $100^{\circ} \mathrm{C}$ durante 48 horas. Luego se realizó la rampa de temperatura en mufla desde los $100^{\circ} \mathrm{C}$ hasta $500^{\circ} \mathrm{C}, 5$ horas, y las cenizas blancas se disolvieron en $50 \mathrm{ml}$ de $\mathrm{HCl}$ concentrado más $100 \mathrm{ml}$ de $\mathrm{H}_{2} \mathrm{O}$ desionizada. Se prepararon soluciones estándares (AOAC 986.15). Los forrajes, secados en estufa, fueron procesados hasta cenizas. La calcinación se efectuó a 
Tabla 1. Macro y microminerales en suero de vacas lecheras en distintos estadios fisiológicos $(\overline{\mathrm{x}} \pm \mathrm{DE})$.

\begin{tabular}{|c|c|c|c|c|c|c|c|}
\hline \multirow[b]{2}{*}{ estado fisiológico } & \multicolumn{4}{|c|}{ macrominerales $(\mathrm{n}=180)$} & \multicolumn{3}{|c|}{ microminerales $(\mathrm{n}=180)$} \\
\hline & $\begin{array}{c}\mathrm{Na} \\
\mathrm{mmol} / 1\end{array}$ & $\begin{array}{c}\mathrm{K} \\
\mathrm{mmol} / 1\end{array}$ & $\begin{array}{c}\mathrm{Ca} \\
\mathrm{mg} / \mathrm{dl}\end{array}$ & $\underset{\mathrm{mg} / \mathrm{dl}}{\mathrm{Mg}}$ & $\begin{array}{c}\mathrm{Cu} \\
\mathrm{mg} / 1\end{array}$ & $\underset{\mathrm{mg} / 1}{\mathrm{Zn}}$ & $\begin{array}{l}\mathrm{Fe} \\
\mathrm{mg} / 1\end{array}$ \\
\hline \multicolumn{8}{|l|}{ gestación } \\
\hline campo A & $\begin{array}{l}133,055 \\
\pm 1,794\end{array}$ & $\begin{array}{l}5,176 \mathrm{a} \\
\pm 0,404\end{array}$ & $\begin{array}{l}8,980 \mathrm{a} \\
\pm 1,030\end{array}$ & $\begin{array}{l}1,745 \mathrm{a} \\
\pm 0,217\end{array}$ & $\begin{array}{l}0,533 \mathrm{a} \\
\pm 0,118\end{array}$ & $\begin{array}{l}0,373 \mathrm{a} \\
\pm 0,070\end{array}$ & $\begin{array}{l}1,167 \mathrm{a} \\
\pm 0,202\end{array}$ \\
\hline campo B & $\begin{array}{l}131,496 \\
\pm 3,181\end{array}$ & $\begin{array}{c}5,125 \\
\pm 1,617\end{array}$ & $\begin{array}{l}9,227 \mathrm{a} \\
\pm 0,595\end{array}$ & $\begin{array}{l}1,720 \mathrm{a} \\
\pm 0,239\end{array}$ & $\begin{array}{l}0,580 \mathrm{a} \\
\pm 0,132\end{array}$ & $\begin{array}{l}0,360 \mathrm{a} \\
\pm 0,099\end{array}$ & $\begin{array}{c}1,473 \\
\pm 0,483\end{array}$ \\
\hline campo C & $\begin{array}{c}133,055 \mathrm{a} \\
\pm 1,794 \\
\end{array}$ & $\begin{array}{l}5,176 \mathrm{a} \\
\pm 0,404 \\
\end{array}$ & $\begin{array}{l}8,980 \mathrm{a} \\
\pm 1,030 \\
\end{array}$ & $\begin{array}{l}1,747 \mathrm{a} \\
\pm 0,217 \\
\end{array}$ & $\begin{array}{l}0,533 \mathrm{a} \\
\pm 0,118 \\
\end{array}$ & $\begin{array}{l}0,411 \text { a } \\
\pm 0,072 \\
\end{array}$ & $\begin{array}{l}1,167 \mathrm{a} \\
\pm 0,202 \\
\end{array}$ \\
\hline \multicolumn{8}{|l|}{ preparto } \\
\hline campo A & $\begin{array}{r}130,412 \\
\pm 1,916\end{array}$ & $\begin{array}{l}5,210 \mathrm{a} \\
\pm 0,500\end{array}$ & $\begin{array}{c}10,360 \mathrm{~b} \\
\pm 1,505\end{array}$ & $\begin{array}{l}2,193 \mathrm{~b} \\
\pm 0,198\end{array}$ & $\begin{array}{l}0,760 \mathrm{~b} \\
\pm 0,106\end{array}$ & $\begin{array}{c}0,760 \mathrm{~b} \\
\pm 0,118\end{array}$ & $\begin{array}{l}1,193 \mathrm{a} \\
\pm 0,356\end{array}$ \\
\hline campo B & $\begin{array}{l}133,023 \\
\pm 4,642\end{array}$ & $\begin{array}{c}5,231 \\
\pm 0,374\end{array}$ & $\begin{array}{l}9,160 \mathrm{a} \\
\pm 0,582\end{array}$ & $\begin{array}{l}2,120 \mathrm{~b} \\
\pm 0,194\end{array}$ & $\begin{array}{l}0,633 \mathrm{a} \\
\pm 0,072\end{array}$ & $\begin{array}{l}0,940 \mathrm{~b} \\
\pm 0,176\end{array}$ & $\begin{array}{c}1,173 \\
\pm 0,306\end{array}$ \\
\hline campo C & $\begin{array}{c}147,925 \mathrm{~b} \\
\pm 8,892\end{array}$ & $\begin{array}{l}4,674 \mathrm{~b} \\
\pm 0,587\end{array}$ & $\begin{array}{c}11,233 \mathrm{~b} \\
\pm 3,134\end{array}$ & $\begin{array}{l}2,447 \mathrm{~b} \\
\pm 0,196\end{array}$ & $\begin{array}{l}0,740 \mathrm{~b} \\
\pm 0,130\end{array}$ & $\begin{array}{l}0,747 \mathrm{~b} \\
\pm 0,167 \\
\end{array}$ & $\begin{array}{l}1,273 \mathrm{a} \\
\pm 0,347 \\
\end{array}$ \\
\hline \multicolumn{8}{|l|}{ posparto } \\
\hline campo A & $\begin{array}{l}130,635 \\
\pm 1,871\end{array}$ & $\begin{array}{l}4,767 \mathrm{~b} \\
\pm 0,658\end{array}$ & $\begin{array}{l}9,793 \mathrm{~b} \\
\pm 1,533\end{array}$ & $\begin{array}{l}2,040 \mathrm{~b} \\
\pm 0,334\end{array}$ & $\begin{array}{l}0,693 \mathrm{~b} \\
\pm 0,110\end{array}$ & $\begin{array}{l}0,693 \mathrm{~b} \\
\pm 0,110\end{array}$ & $\begin{array}{l}0,960 \mathrm{~b} \\
\pm 0,159\end{array}$ \\
\hline campo B & $\begin{array}{l}131,032 \\
\pm 3,508\end{array}$ & $\begin{array}{c}5,174 \\
\pm 0,442\end{array}$ & $\begin{array}{l}8,987 \mathrm{~b} \\
\pm 0,843\end{array}$ & $\begin{array}{l}1,947 \mathrm{~b} \\
\pm 0,210\end{array}$ & $\begin{array}{l}0,640 \mathrm{a} \\
\pm 0,083\end{array}$ & $\begin{array}{l}0,833 \mathrm{~b} \\
\pm 0,180\end{array}$ & $\begin{array}{c}1,173 \\
\pm 0,252\end{array}$ \\
\hline campo C & $\begin{array}{c}150,177 \mathrm{~b} \\
\pm 7,068\end{array}$ & $\begin{array}{l}3,918 \mathrm{~b} \\
\pm 0,599\end{array}$ & $\begin{array}{c}10,887 \mathrm{~b} \\
\pm 2,036\end{array}$ & $\begin{array}{l}2,433 \mathrm{~b} \\
\pm 0,238\end{array}$ & $\begin{array}{l}0,753 \mathrm{~b} \\
\pm 0,125\end{array}$ & $\begin{array}{l}0,761 \mathrm{~b} \\
\pm 0,116\end{array}$ & $\begin{array}{l}1,540 \mathrm{~b} \\
\pm 0,336\end{array}$ \\
\hline \multicolumn{8}{|l|}{ lactación } \\
\hline campo A & $\begin{array}{l}130,362 \\
\pm 1,714\end{array}$ & $\begin{array}{l}4,687 \mathrm{~b} \\
\pm 0,477\end{array}$ & $\begin{array}{c}9,110 \mathrm{a} \\
\pm 1,14\end{array}$ & $\begin{array}{l}2,04 \mathrm{~b} \\
\pm 0,15\end{array}$ & $\begin{array}{l}0,620 \mathrm{a} \\
\pm 0,068\end{array}$ & $\begin{array}{l}0,687 \mathrm{~b} \\
\pm 0,106\end{array}$ & $\begin{array}{l}0,893 \mathrm{~b} \\
\pm 0,133\end{array}$ \\
\hline campo B & $\begin{array}{l}129,365 \\
\pm 2,079\end{array}$ & $\begin{array}{c}5,00 \\
\pm 0,422\end{array}$ & $\begin{array}{c}8,60 \mathrm{~b} \\
\pm 0,582\end{array}$ & $\begin{array}{l}1,893 \mathrm{a} \\
\pm 0,240\end{array}$ & $\begin{array}{l}0,720 \mathrm{~b} \\
\pm 0,094\end{array}$ & $\begin{array}{c}0,867 \mathrm{~b} \\
\pm 0,168\end{array}$ & $\begin{array}{c}1,013 \\
\pm 0,160\end{array}$ \\
\hline campo C & $\begin{array}{c}129,441 \mathrm{a} \\
\pm 2,319\end{array}$ & $\begin{array}{l}5,002 \mathrm{a} \\
\pm 0,259\end{array}$ & $\begin{array}{l}9,113 \mathrm{a} \\
\pm 0,892\end{array}$ & $\begin{array}{l}1,893 \mathrm{a} \\
\pm 0,158\end{array}$ & $\begin{array}{l}0,760 \mathrm{~b} \\
\pm 0,112\end{array}$ & $\begin{array}{l}0,711 \mathrm{~b} \\
\pm 0,085\end{array}$ & $\begin{array}{l}0,853 \mathrm{a} \\
\pm 0,155\end{array}$ \\
\hline
\end{tabular}

Valores con letras distintas en la misma columna difieren estadísticamente $(\mathrm{p}<0,05)$ en bovinos del mismo campo.

una temperatura de $550^{\circ} \mathrm{C}$. Las muestras de alfalfa se obtuvieron de distintos puntos de la superficie total de pastoreo, a la altura que come el animal. El pasto se colocó en bolsas de nylon correctamente rotuladas para su identificación. Entre la recolección y transporte las muestras fueron guardadas en una conservadora con hielo. Para la determinación de los minerales en el forraje, se prepararon soluciones empleando las técnicas AOAC 968.08.

Los estudios del perfil mineral se efectuaron en un espectrofotómetro de absorción y emisión atómica de llama Perkin Elmer, modelo 3110, para los minerales cobre $(\mathrm{Cu})$, hierro $(\mathrm{Fe})$, cinc $(\mathrm{Zn})$, calcio $(\mathrm{Ca})$, magnesio $(\mathrm{Mg})$, sodio $(\mathrm{Na})$ y potasio $(\mathrm{K})$. El selenio $(\mathrm{Se})$ se valoró en un espectrofotómetro de absorción atómica de llama con generación de hidruros Perkin Elmer 100. Todas las mediciones se hicieron por triplicado y se consideró el valor promedio en cada una de las determinaciones. Se realizaron determinaciones de P inorgánico por método colorimétrico, en los períodos fisiológicos de posparto y lactación, momentos de gran importancia para el animal comprometido a la producción de leche.

Para el análisis estadístico de los datos, los valores obtenidos se tabularon categóricamente en planillas de cálculo (Excel) y se procesaron mediante el software Infostat. Se obtuvieron estadísticas descriptivas para- métricas (media $\bar{x}$, desvío estándar DE). Las variables respuestas (niveles de los minerales) fueron analizadas según un modelo mixto, que incluyó el efecto de los campos, los períodos fisiológicos y la interacción entre ambos factores. Las pruebas se realizaron considerando un nivel de probabilidad del $5 \%$.

\section{RESULTADOS Y DISCUSIÓN}

Los valores de los minerales en suero, para los cuatro estados fisiológicos en los tres campos, se muestran en la Tabla 1. Debido a que la interacción campo $x$ periodo fue significativa $(\mathrm{p}<0,05)$, se analizaron los valores promedios de los minerales en suero a lo largo de los distintos períodos fisiológicos, dentro de cada establecimiento.

Los valores medios de Ca en gestación y lactación en los tres campos, y el preparto y posparto del campo $\mathrm{B}$, fueron inferiores al rango de referencia de la literatura ${ }^{4}$. La normocalcemia detectada en el preparto de los campos A y C debería relacionarse con el adecuado ingreso del catión y el buen funcionamiento del mecanismo homeostático ${ }^{12}$. Seguramente por aumentar la exigencia fisiológica, ocurrió deficiencia de $\mathrm{Ca}$ en los períodos de gestación y lactación, que para los campos A y C no fueron significativas ${ }^{12,13}$. En el período 
Tabla 2. Minerales en muestras de leche para los estados fisiológicos de posparto y lactación en vacas de los campos A, B y C $(\overline{\mathrm{x}} \pm \mathrm{DE})$.

\begin{tabular}{|c|c|c|c|c|c|c|c|c|}
\hline \multirow[t]{2}{*}{ campo } & \multirow[t]{2}{*}{ período } & \multicolumn{4}{|c|}{$\begin{array}{l}\text { macrominerales }(\mathrm{n}=90) \\
\mathrm{mg} / 100 \mathrm{~g}\end{array}$} & \multicolumn{3}{|c|}{$\begin{array}{l}\underset{\mathrm{mg} / \mathrm{l}}{\operatorname{microminerales}(\mathrm{n}=90)} \\
\end{array}$} \\
\hline & & $\mathrm{Na}$ & $\mathrm{K}$ & $\mathrm{Ca}$ & $\mathrm{Mg}$ & $\mathrm{Cu}$ & $\mathrm{Zn}$ & $\mathrm{Fe}$ \\
\hline \multirow{2}{*}{ A } & $\begin{array}{l}\text { posparto } \\
\mathrm{n}=15\end{array}$ & $\begin{array}{r}48,57 \\
\pm 7,74\end{array}$ & $\begin{array}{l}160,99 \mathrm{a} \\
\pm 12,244\end{array}$ & $\begin{array}{c}119,21 \\
\pm 13,42\end{array}$ & $\begin{array}{l}7,73 \mathrm{a} \\
\pm 1,32\end{array}$ & $\begin{array}{l}0,160 \\
\pm 0,06\end{array}$ & $\begin{array}{c}2,78 \\
\pm 0,35\end{array}$ & $\begin{array}{l}0,324 \mathrm{a} \\
\pm 0,024\end{array}$ \\
\hline & $\begin{array}{l}\text { lactación } \\
\mathrm{n}=15\end{array}$ & $\begin{array}{l}48,76 \\
\pm 4,37 \\
\end{array}$ & $\begin{array}{l}153,56 \mathrm{~b} \\
\pm 14,29 \\
\end{array}$ & $\begin{array}{l}113,55 \\
\pm 6,53 \\
\end{array}$ & $\begin{array}{l}10,44 \mathrm{~b} \\
\pm 3,85\end{array}$ & $\begin{array}{l}0,175 \\
\pm 0,07\end{array}$ & $\begin{array}{c}2,92 \\
\pm 0,48 \\
\end{array}$ & $\begin{array}{l}0,403 \mathrm{~b} \\
\pm 0,066 \\
\end{array}$ \\
\hline \multirow{2}{*}{ B } & $\begin{array}{l}\text { posparto } \\
\mathrm{n}=15\end{array}$ & $\begin{array}{l}46,77 \mathrm{a} \\
\pm 3,48\end{array}$ & $\begin{array}{l}167,93 \mathrm{a} \\
\pm 12,62\end{array}$ & $\begin{array}{l}128,39 \mathrm{a} \\
\pm 15,76\end{array}$ & $\begin{array}{c}8,83 \\
\pm 1,77\end{array}$ & $\begin{array}{l}0,199 \mathrm{a} \\
\pm 0,10\end{array}$ & $\begin{array}{l}3,15 \mathrm{a} \\
\pm 0,83\end{array}$ & $\begin{array}{l}0,373 \mathrm{a} \\
\pm 0,074\end{array}$ \\
\hline & $\begin{array}{l}\text { lactación } \\
\mathrm{n}=15\end{array}$ & $\begin{array}{l}51,08 \mathrm{~b} \\
\pm 8,74\end{array}$ & $\begin{array}{l}157,43 \mathrm{~b} \\
\pm 8,33\end{array}$ & $\begin{array}{c}110,00 \mathrm{~b} \\
\pm 8,92\end{array}$ & $\begin{array}{c}7,74 \\
\pm 0,75\end{array}$ & $\begin{array}{l}0,163 \mathrm{~b} \\
\pm 0,06\end{array}$ & $\begin{array}{l}2,44 \mathrm{~b} \\
\pm 0,45\end{array}$ & $\begin{array}{l}0,443 \mathrm{~b} \\
\pm 0,133\end{array}$ \\
\hline \multirow{2}{*}{$\mathrm{C}$} & $\begin{array}{l}\text { posparto } \\
\mathrm{p}=15\end{array}$ & $\begin{array}{l}47,28 \\
\pm 8,98\end{array}$ & $\begin{array}{l}155,57 \mathrm{a} \\
\pm 18,8\end{array}$ & $\begin{array}{l}115,53 \\
\pm 11,01\end{array}$ & $\begin{array}{c}7,16 \\
\pm 1,42\end{array}$ & $\begin{array}{l}0,169 \\
\pm 0,06\end{array}$ & $\begin{array}{c}2,64 \\
\pm 0,46\end{array}$ & $\begin{array}{l}0,335 \mathrm{a} \\
\pm 0,039\end{array}$ \\
\hline & $\begin{array}{l}\text { lactación } \\
\mathrm{n}=15\end{array}$ & $\begin{array}{l}48,95 \\
\pm 4,01\end{array}$ & $\begin{array}{c}150,31 \mathrm{~b} \\
\pm 11,51\end{array}$ & $\begin{array}{l}112,03 \\
\pm 6,39\end{array}$ & $\begin{array}{l}7,35 \\
\pm 0,91\end{array}$ & $\begin{array}{l}0,188 \\
\pm 0,07\end{array}$ & $\begin{array}{c}2,77 \\
\pm 0,49\end{array}$ & $\begin{array}{l}0,420 \mathrm{~b} \\
\pm 0,076\end{array}$ \\
\hline
\end{tabular}

Valores con letras distintas en la misma columna difieren estadísticamente $(\mathrm{p}<0,05)$ en bovinos del mismo campo.

de gestación se observó hipomagnesemia significativa $(\mathrm{p}<0,05)$ en los tres campos, quizás relacionada a la depresión del apetito y al estrés del animal próximo al parto ${ }^{7}$. El valor más bajo de $\mathrm{Mg}$ durante la lactación, respecto a la transición, podría deberse a que la investigación se realizó durante el verano, época de calor, con la consiguiente pérdida del mineral por sudoración ${ }^{4}$.

En las etapas de gestación y lactación del campo $\mathrm{C}$ se evidenció hiponatremia significativa $(\mathrm{p}<0,05)$ con respecto al periparto, cuya causa podría atribuirse a la mayor demanda de este macromineral para la síntesis de leche. Las diferencias significativas $(\mathrm{p}<0,05)$ registradas para $\mathrm{Ca}$ y $\mathrm{Na}$ en la gestación respecto del preparto en el campo C, tal vez se deban a que los animales Holstein están más adaptados a las altas temperaturas y a la elevada humedad de la región en la estación investigada $^{5,14}$.

En la transición se observaron disminuciones en los valores promedios de calcio y magnesio, presentando diferencia significativas $(p<0,05)$ el calcio del rodeo $\mathrm{B}$, que podría deberse a que días previos al parto comienza la síntesis de calostro, y también se debe considerar que en estos períodos el mecanismo regulatorio homeostático está ausente ${ }^{5,13}$. Cabe destacar que durante la transición, en los rodeos Holstein-Friesian de los campos A y B, se obtuvieron valores promedios inferiores a los referenciales para los minerales calcio, magnesio y sodio, registrándose una diferencia significativa en los valores de $\mathrm{Ca}$ en el campo B. Ello puede estar relacionado con la raza pura de los animales, quienes poseen menor contenido sérico de $\mathrm{Ca}, \mathrm{Mg}$ y $\mathrm{Na}^{5}$. Los valores medios de potasio oscilaron dentro del intervalo de referencia ${ }^{4}$ en todos los campos y períodos fisiológicos. La diferencia significativa $(p<0,05)$ observada para el $\mathrm{K}$ durante el periparto con respecto a la lactación en el campo $\mathrm{C}$, se debería fundamentalmente a los distintas cantidades y tipos de alimentos suministrados.

En los campos A y C los valores promedios de cobre en suero se encontraron en el límite inferior del valor referencial ${ }^{17}$ solo durante la gestación, lo cual indicaría que en los demás períodos las reservas hepáticas de este catión les permitieron mantener sus niveles plasmáticos dentro del rango normal ${ }^{12}$. Los valores de Fe en suero permanecieron por debajo del rango de referencia ${ }^{17}$ durante la lactación de los tres rodeos y en el posparto-lactación de los campos A y B, circunstancia que pudo deberse al estrés y a las altas temperaturas del verano ${ }^{10}$, al igual que los valores promedios del cinc por debajo del rango de referencia ${ }^{17}$ en los cuatro estados fisiológicos, de los campos A y C. En la lactación, los bajos valores de $\mathrm{Zn}$ en los campos A y $\mathrm{C}(\mathrm{p}<0,05)$, asociados a la gestación, se deberían a la elevada demanda del mineral durante la secreción de leche ${ }^{20}$.

La determinación de selenio se realizó en 10 muestras de suero en cada campo solo en el posparto por ser una etapa productiva de alta exigencia, momento en que se manifiesta la deficiencia. Los valores promedios y desvíos estándares fueron $26,68 \pm 3,51 ; 32,67 \pm 4,62$ y $57,50 \pm 12,02 \mathrm{ug} / \mathrm{l}$ en los campos A, B y C, respectivamente, concentraciones que no se apartan del rango normal ${ }^{18}$. Los valores de fósforo inorgánico en suero de los dos períodos fisiológicos (posparto y lactación) fueron $5,96 \pm 0,86-5,18 \pm 0,75 ; 6,89 \pm 1,45-6,21 \pm$

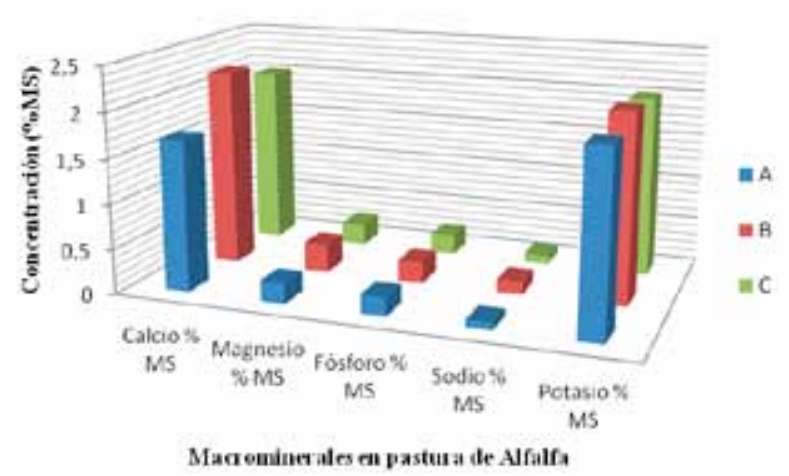

Figura 1. Valores medios de macrominerales en pastura de alfalfa de los campos A, B y C $(n=2)$, expresados en porcentaje de materia seca (MS). 
2,$64 ; 5,78 \pm 1,12-5,10 \pm 1,33 \mathrm{mg} / \mathrm{dl}$ para los campos A, B y C. Todos los valores se encontraron dentro del rango de referencia ${ }^{3}$ y se registraron diferencias estadísticas dentro del mismo período entre los campos $\mathrm{A}$ versus $B$ y entre los campos $C$ versus $B(p<0,05)$.

En la Tabla 2 se presentan los valores minerales obtenidos en muestras de leche. En ambos estados fisiológicos, los promedios de $\mathrm{Na}, \mathrm{Mg}, \mathrm{Fe}$ y $\mathrm{Zn}$ estuvieron por debajo del rango de referencia ${ }^{19}$. El aumento significativo $(\mathrm{p}<0,05)$ observado en el valor medio del $\mathrm{Mg}$ desde el posparto a la lactación avanzada en el campo A, se debería al déficit forrajero invernal ${ }^{1}$. Se registraron diferencias estadísticas $(p<0,05)$ en los valores de $\mathrm{Ca}$ con tendencia a descender en el campo B, que se podrían explicar como consecuencia de la mayor demanda del macromineral en la composición de la leche ${ }^{19}$. Teniendo en cuenta los valores de referencia del $\mathrm{Na}$ en leche de la cuenca centro de Santa Fe, los mismos se encontraron por debajo del valor medio, significativamente entre los períodos en el campo B. En la lactación se registró una disminución $(\mathrm{p}<0,05)$ de los valores de magnesio en el campo B y del potasio $(p<0,05)$ en los 3 campos. El incremento significativo de sodio en leche en el campo B, puede haberse debido a un desequilibrio mineral en el metabolismo ${ }^{19}$.

El cobre en leche se encontró dentro de los niveles de referencia. Los valores medios de cinc determinados en muestras de leche, en ambos períodos para los campos B y C, se encontraron por debajo del valor de referencia ${ }^{6}$. Se observó descenso significativo $(\mathrm{p}<0,05)$ en los valores promedios de $\mathrm{Zn}$ en muestras de leche, desde el posparto hacia la lactación en el campo $\mathrm{B}^{12}$. Las disminuciones de los valores promedios de cinc y magnesio en la leche de los animales del campo B, y el descenso del calcio y potasio $(\mathrm{p}<0,05)$ observado en los tres rodeos hacia los 60 días de la lactación, se podrían justificar teniendo en cuenta un posible efecto de dilución de los minerales en la leche ${ }^{6,16}$.

En la Figura 1 se muestran los promedios de algunos macrominerales en la alfalfa. Se advierte que los valores más altos correspondieron al campo B para las determinaciones de calcio $(2,22 \% \mathrm{MS})$, fósforo $(0,24 \%$ MS), sodio ( $0,15 \% \mathrm{MS})$, potasio $(2,1 \% \mathrm{MS})$ y magnesio $(0,32 \% \mathrm{MS})$, siendo este último diferente $(\mathrm{p}<0,05)$ con respecto al registrado en los campos A y C. La mayoría de los forrajes contenían niveles de $\mathrm{Mg}$ superiores a los requeridos por los animales.

En la MS de alfalfa consumida por los bovinos, se observaron diferencias estadísticas en cinc, molibdeno y cobre en los distintos campos. Los valores medios de Zn fueron: 28, 22 y $25 \mathrm{mg} / \mathrm{kg}$; los de Mo: 2,0 , 2,5 y 2,6 mg/kg; los de Cu: 13, 11 y $10 \mathrm{mg} / \mathrm{kg}$, y los de hierro: 226,510 y $358 \mathrm{mg} / \mathrm{kg}$ para los campos A, B y C respectivamente. Los minerales en verdeos de invierno y verano (MS), libres de sustancias químicas, revelaron valores de: calcio $(0,31 \pm 0,09$ y $0,25 \pm 0,03 \%)$, magnesio $(0,26 \pm 0,31$ y $0,24 \pm 0,02 \%)$, fósforo $(0,20 \pm 0,05$ y $0,18 \pm 0,04 \%)$, sodio $(0,87 \pm 1,28$ y $0,99 \pm 1,29 \%)$, potasio $(2,3 \pm 0,68$ y $2,5 \pm 0,69 \%)$, hierro $(1068 \pm 459,54$ y
$1142 \pm 491,38 \mathrm{mg} / \mathrm{kg})$, cinc $(29,5 \pm 11,16$ y $34 \pm 8,15 \mathrm{mg} / \mathrm{kg})$ y cobre $(13 \pm 4,52$ y $10,2 \pm 5,31 \mathrm{mg} / \mathrm{kg})$ para los campos A y B respectivamente, siendo las duplas de los microminerales $\mathrm{Fe}, \mathrm{Zn}$ y $\mathrm{Cu}$ significativamente diferentes entre sí $(\mathrm{p}<0,05)$.

Los valores medios de molibdeno en la alfalfa se encontraron dentro del límite superior del rango de referencia ${ }^{16}$. Tal circunstancia plantea una posible competencia de tipo antagonista entre Mo y $\mathrm{Zn}$ dietarios, que explicaría los bajos valores de $\mathrm{Zn}$ en el suero de las vacas $(p<0,05)$. En esta investigación, los bajos niveles de cinc en la leche se podrían atribuir a que en la alfalfa el oligoelemento se encuentra por debajo del requerimiento mínimo para los bovinos, como fue observado en los tres rodeos. Los valores bajos de cinc en la gestación $(\mathrm{p}<0,05)$ tendrían relación con la etapa invernal de muestreo, sumado a que la mayoría de los alimentos para los rumiantes son escasos en dicho oligoelemento, lo cual causaría desbalances en el animal ${ }^{15}$. Se afirma que un aporte inferior a $45 \mathrm{mg} / \mathrm{kg}$ de $\mathrm{Zn}$ en la dieta podría provocar una carencia del micromineral en el ganado vacuno, debido a que la mayoría de los alimentos para los rumiantes son deficientes en cinc ${ }^{20}$. El incremento del valor promedio de molibdeno no produjo interferencia en la absorción de cobre, situación observada a partir del valor de cobre sérico normal. Los valores medios del cobre en alfalfa y verdeos se encontraron por encima de su valor de corte $(>10 \mathrm{mg} / \mathrm{kg})$, lo cual explica los valores normales de $\mathrm{Cu}$ observados en suero y leche. La relación cobre-molibdeno en los alimentos fue normal, por lo cual puede concluirse que no existió interferencia en la absorción de cobre.

A partir de la caracterización del perfil mineral en suero de los bovinos lecheros de la región centro de Santa Fe, se concluye que aunque algunos minerales se encuentran desfasados del rango de referencia considerado para compararlos, no se observaron manifestaciones clínicas de patologías relacionadas con la carencia de los mismos. Si bien los valores de $\mathrm{Mg}$, $\mathrm{Na}, \mathrm{Zn}$ y Fe fueron bajos en leche, ello fue un reflejo de las bajas concentraciones de dichos minerales en las muestras de suero. Por otra parte, los valores encontrados en suero y leche en los cuatro períodos fisiológicos aportan información valiosa acerca del perfil mineral, que podría ser tenida en cuenta por productores y profesionales dedicados a la asistencia técnica de tambos de la región.

\section{REFERENCIAS}

1. Andrews AH. 2005. Sanidad del ganado vacuno lechero, Acribia, Zaragoza, p.70-71.

2. Association of Official Analytical Chemist, AOAC. 1990. Official methods of analysis. Agricultural chemicals; contaminants, Arlington (USA), $1.117 \mathrm{p}$.

3. Bavera GA. 2006. Suplementación mineral con nitrógeno no proteico del bovino a pastoreo. On line: http://www. produccion-animal.com.ar (Río Cuarto, Argentina). p.21101. 
4. Buffarini MA, Minatel L, Cseh S. 2008. Variaciones estacionales de minerales en sangre en dos rodeos de cría en pastoreo en General Villegas, Memoria Técnica 20072008 INTA Gral. Villegas, p. 88-92.

5. Campos RG, Cubillo C, Rodas AG. 2007. Indicadores metabólicos en razas lecheras especializadas en condiciones tropicales en Colombia. Acta Agron 56: 2-10.

6. Closa SJ, De Landeta MC, Andérica D, Pighín A. 2003. Contenido de nutrientes minerales en leche de vaca y derivados de Argentina. Arch Latin Nutr 53: 3-8.

7. Contreras PA, Valenzuela F, Wittwer H, Böhmwald H. 1996. Desbalances metabólicos nutricionales más frecuentes en rebaños de pequeños productores de leche en Valdivia, Chile. Arch Med Vet 28: 39-50.

8. Coppo JA, Coppo NB, Koza GA, Slanac AL. 1999. Indicadores nutricionales en la hacienda de áreas inundadas de las provincias de Corrientes, Chaco y Formosa. Actas Cien \& Técn UNNE (Argentina) 4: 117-120.

9. Corbellini CN. 1998. Influencia de la nutrición en las enfermedades de la producción de las vacas lecheras en transición. Proyecto Lechero INTA Pergamino (Argentina), http://www.sinervia.com/library, $25 \mathrm{p}$.

10. Drugueri L. 2000. El diagnóstico y evaluación de la deficiencia de cinc en los bovinos. On line: www.zoetecnocampo.com.www.zoetecnocampo.com/Documentos / zinc_bovinos.htm, p. 11-14.

11. Fader OW. 2001. Los minerales en la nutrición y la salud animal en la región central de la Provincia de Córdoba. On line: http://www.produccion-animal.com.ar.

12. Freer M, Dove H, Nolan JV. 2007. Nutrient requirements of domesticated ruminants, CSIRO Publishing, Melbourne, p.115-169.
13. González. FH. 2000. Indicadores sangüíneos do metabolismo mineral em rumiantes. In: Perfil metabólico em ruminantes: seu uso em nutrição e doenças nutricionais (Ospina H, Ribeiro LA, Ed.), Gráfica da UFRGS, Porto Alegre, p. 31-49.

14. Leiva PE, García MS, Vélez MA, Valtorta SE. 2005. Respuestas fisiológicas de vacas Holando Argentino y cruza Jersey Holando en la cuenca lechera santafesina. Rev FAVE Cs Agr 4: 1-2. www.fca.unl.edu.ar/pages/investigacion/revista-fave.php.

15. Minatel L, Buffarini MA, Scarlata EF, Dallorso ME, Carfagnini JC. 2004. Niveles de cobre, hierro, zinc y selenio de bovinos del noroeste de la Provincia de Buenos Aires. Rev Arg Prod Anim 24: 225-235.

16. Morales Almaráz E, Domínguez I, González M. 2007. Diagnóstico mineral en forraje y suero sanguíneo de bovinos lecheros en dos épocas en el valle central de México. Téc Pecu Méx 45: 329-344.

17. Rosa DE, Mattioli GA. 2002. Metabolismo y deficiencia de cobre en los bovinos. Analecta Vet 22: 7-16.

18. Silva JH, Quiroga MA, Auza NJ. 2000. Selenio en el rumiante. Relaciones suelo, planta, animal. Med Vet 17: 229-238.

19. Taverna M, Chávez M, Páez R, Cuatrín A, Negri L. 2008. Caracterización de la aptitud tecnológica de la leche destinada a la elaboración de leche en polvo entera en la cuenca lechera central. Rev Tecn Láct Latinoam 53: 40-43.

20. Underwood EJ, Suttle NF. 1999. Mineral nutrition of livestock, 3rd ed., CAB International, Edinburgh (UK) p. 456-463. 